\title{
Comparative Studies to Solve Compressible Problems by Multiscale Finite Element Methods
}

\author{
Sérgio Souza Bento ${ }^{1}$ \\ Ramoni Zancanela Sedano ${ }^{2}$ \\ Leonardo Muniz de Lima ${ }^{3}$ \\ Lucia Catabriga ${ }^{4}$ \\ High Performance Computing Lab, UFES, Vitória, ES \\ Isaac P. Santos ${ }^{5}$ \\ Department of Applied Mathematics, UFES, São Mateus, ES
}

\begin{abstract}
In this work we evaluate two multiscale methodologies to solve compressible flow problems, named, Dynamic Diffusion (DD) and Nonlinear Multiscale Viscosity (NMV), using the well know predictor-multicorrector time integration scheme. The subgrid scale space is defined using bubble functions whose degrees of freedom are locally eliminated in favor of the degrees of freedom that live on the resolved scales. The time integration schemes assume that the resolved coarse scale advances in time by second order approximation and the unresolved scale can advance by first and second order approximations. Performance and accuracy comparisons are conducted based on benchmark 2D problems.
\end{abstract}

Keywords. Finite Element Method, Compressible Flows, Multiscale Stabilized Formulation

\section{Introduction}

Numerical solutions of the compressible flows may exhibit global spurious oscillations, especially near shock regions. More accurate and stable results can be obtained considering variational multiscale formulations [1]. In a general sense, each of these alternative methodologies are based on adding some type of stabilization to the standard Galerkin formulation and usually depends on the definition of one or more tuning parameters that usually play crucial role on the accuracy of the final solution.

Arruda et al. [2] presented a nonlinear multiscale method, named Dynamic Diffusion (DD), to solve advection dominated transport problem, where the subgrid space is constructed by bubbles functions defined into elements and the amount of nonlinear diffusion is similar to the method presented in [3], but adding in both scales (subgrid and coarse) of the discretization. This methodology was extended to the compressible Euler equations

\footnotetext{
${ }^{1}$ sergio.bento@ufes.br

${ }^{2}$ rsedano@inf.ufes.br

${ }^{3}$ lmuniz@ifes.edu.br

${ }^{4}$ luciac@inf.ufes.br

${ }^{5}$ isaac.santos@ufes.br
} 
in [4] and recently, it was improved by [5] and renamed the Nonlinear Multiscale Viscosity (NMV) method. The basic idea of the NMV method, as the DD method, is to add a nonlinear artificial viscosity in all scales of the discretization, but the amount of artificial viscosity is defined by the stabilization parameter of the $\mathrm{YZ} \beta$ method, as proposed in [6].

The remainder of this work is organized as follows. Section 2 briefly addresses the governing equations and the variational multiscale formulation. Numerical experiments are conducted in Section 3 to show the behavior of the multiscale finite element methods for two-dimensional test problems for the Euler equations. Section 4 concludes this paper.

\section{Governing Equations and Variational Multiscale Formu- lation}

The two-dimensional Euler equations in conservative variables, $\mathbf{U}=(\rho, \rho u, \rho v, \rho e)$, without source terms are an inviscid system of conservation laws represented by

$$
\frac{\partial \mathbf{U}}{\partial t}+\mathbf{A}_{\mathbf{x}} \frac{\partial \mathbf{U}}{\partial x}+\mathbf{A}_{\mathbf{y}} \frac{\partial \mathbf{U}}{\partial y}=\mathbf{0}, \quad \text { on } \Omega \times\left[0, T_{f}\right],
$$

where $\rho$ is the fluid density, $\mathbf{u}=(u, v)$ is the velocity vector, $e$ is the total energy per unit mass, $\mathbf{A}_{x}=\frac{\partial \mathbf{F}_{x}}{\partial \mathbf{U}}$ and $\mathbf{A}_{y}=\frac{\partial \mathbf{F}_{y}}{\partial \mathbf{U}}$ where $\mathbf{F}_{x}$ and $\mathbf{F}_{y}$ are the Euler fluxes, $\Omega$ is a domain in $\mathbb{R}^{2}$, and $T_{f}$ is a positive real number, representing the final time. Associated to Eq. (1) we have a proper set of boundary and initial conditions.

To define the finite element discretization, we consider a triangular partition $\mathcal{T}_{H}$ of the domain $\Omega$ into $n_{e l}$ elements, where: $\Omega=\bigcup_{e=1}^{n_{e l}} \Omega_{e}$ and $\Omega_{i} \cap \Omega_{j}=\emptyset, i, j=1,2, \cdots, n_{e l}$, $i \neq j$. We introduce the space $\mathcal{V}_{E}$, that is written as the direct sum, $\mathcal{V}_{E}=\mathcal{V}_{h} \oplus \mathcal{V}_{B}$, where the subspaces $\mathcal{V}_{h}$ and $\mathcal{V}_{B}$ are given by

$$
\begin{aligned}
& \mathcal{V}_{h}=\left\{\mathbf{U}_{h} \in\left[H^{1}(\Omega)\right]^{4} \quad\left|\quad \mathbf{U}_{h}\right|_{\Omega_{e}} \in\left[\mathbb{P}_{1}\left(\Omega_{e}\right)\right]^{4}, \mathbf{U}_{h} \cdot \mathbf{e}_{k}=g_{k}(t) \text { in } \Gamma_{g_{k}}\right\} ; \\
& \mathcal{V}_{B}=\left\{\mathbf{U}_{B} \in\left[H_{0}^{1}\left(\mathcal{T}_{H}\right)\right]^{4} \quad\left|\quad \mathbf{U}_{B}\right|_{\Omega_{e}} \in\left[\operatorname{span}\left(\psi_{B}\right)\right]^{4}, \quad \forall \Omega_{e} \in \mathcal{T}_{H}\right\},
\end{aligned}
$$

where $\mathbb{P}_{1}\left(\Omega_{e}\right)$ represents the set of first order polynomials in $\Omega_{e}, \psi_{B}$ is a bubble function $\left(0 \leq \psi_{B} \leq 1\right.$ and $\left.\psi_{B} \in H_{0}^{1}\left(\mathcal{T}_{H}\right)\right)$ and $H^{1}, H_{0}^{1}$ are Hilbert spaces. The space $\mathcal{V}_{h}$ represents the resolved (coarse) scale space whereas $\mathcal{V}_{B}$ stands for the subgrid (fine) scale space (Fig. $1)$.

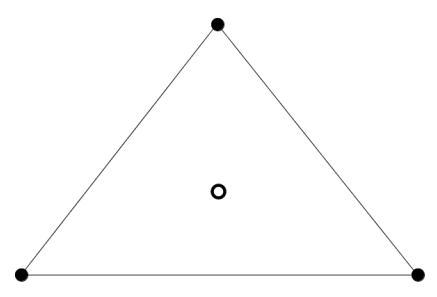

Figure 1: $\mathcal{V}_{E}$ Representation: $\bullet$ stands for $\mathcal{V}_{h}$ nodes and $\circ$ stands for $\mathcal{V}_{B}$ nodes. 
Multiscale methods addressed in this paper for the Euler equation consists of find $\mathbf{U}_{E}=\mathbf{U}_{h}+\mathbf{U}_{B} \in \mathcal{V}_{E}$ with $\mathbf{U}_{h} \in \mathcal{V}_{h}, \mathbf{U}_{B} \in \mathcal{V}_{B}$ such that

$$
\begin{gathered}
\int_{\Omega} \mathbf{W}_{E} \cdot\left(\frac{\partial \mathbf{U}_{E}}{\partial t}+\mathbf{A}_{x}^{h} \frac{\partial \mathbf{U}_{E}}{\partial x}+\mathbf{A}_{y}^{h} \frac{\partial \mathbf{U}_{E}}{\partial y}\right) d \Omega+ \\
\sum_{e=1}^{n e l} \int_{\Omega_{e}} \delta_{h}^{*}\left(\frac{\partial \mathbf{W}_{E}}{\partial x} \cdot \frac{\partial \mathbf{U}_{E}}{\partial x}+\frac{\partial \mathbf{W}_{E}}{\partial y} \cdot \frac{\partial \mathbf{U}_{E}}{\partial y}\right) d \Omega=\mathbf{0}, \quad \forall \mathbf{W}_{E} \in \mathcal{V}_{E},
\end{gathered}
$$

where $\mathbf{W}_{E}=\mathbf{W}_{h}+\mathbf{W}_{B} \in \mathcal{V}_{E}$ with $\mathbf{W}_{h} \in \mathcal{V}_{h}, \mathbf{W}_{B} \in \mathcal{V}_{B}$ and the amount of artificial viscosity is calculated on the element-level. For the DD method inspired by the CAU shock-capturing viscosity parameter [7]:

$$
\delta_{h}^{\mathrm{DD}}=\left\{\begin{array}{l}
\frac{1}{2} \mu(\hbar) \frac{\left\|R\left(\mathbf{U}_{h}\right)\right\|_{\widetilde{A}_{0}^{-1}}}{\left\|\boldsymbol{\nabla}_{\xi} \mathbf{U}_{h}\right\|_{\widetilde{A}_{0}^{-1}}}, \text { if }\left\|\boldsymbol{\nabla} \mathbf{U}_{h}\right\|_{\widetilde{A}_{0}^{-1}}>t o l_{\delta} \\
0, \quad \text { otherwise, }
\end{array}\right.
$$

where $\mu(\hbar)=\sqrt{2 A_{e}}$ is the element length, $A_{e}$ is the element area, $\widetilde{\mathbf{A}}_{0}^{-1}$ is the Jacobian of the transformation between the entropy and conservation variables [8]. For the other hand, the NMV method is defined by considering the YZ $\beta$ shock-capturing viscosity parameter [6]

$$
\delta_{h}^{\mathrm{NMV}}=\left\|\mathbf{Y}^{-1} R\left(\mathbf{U}_{h}\right)\right\|\left(\sum_{i=1}^{n_{s d}}\left\|\mathbf{Y}^{-1} \frac{\partial \mathbf{U}_{h}}{\partial x_{i}}\right\|^{2}\right)^{\frac{\beta}{2}-1}\left\|\mathbf{Y}^{-1} \mathbf{U}_{h}\right\|^{1-\beta}\left(\frac{h}{2}\right)^{\beta},
$$

where

$$
R\left(\mathbf{U}_{h}\right)=\frac{\partial \mathbf{U}_{h}}{\partial t}+\mathbf{A}_{x}^{h} \frac{\partial \mathbf{U}_{h}}{\partial x}+\mathbf{A}_{y}^{h} \frac{\partial \mathbf{U}_{h}}{\partial y}
$$

is the residue of the problem on $\Omega_{e}, \mathbf{Y}$ is a diagonal matrix constructed from the reference values of the components of $\mathbf{U}$, given by $\mathbf{Y}=\operatorname{diag}\left(\left(U_{1}\right)_{\text {ref }},\left(U_{2}\right)_{\text {ref }},\left(U_{3}\right)_{\text {ref }},\left(U_{4}\right)_{\text {ref }}\right), h$ is the local length scale defined as in [6] by

$$
h=2\left(\sum_{a}\left|\mathbf{j} \cdot \nabla N_{a}\right|\right)^{-1}, \text { where } \mathbf{j} \text { is a unit vector defined as } \mathbf{j}=\frac{\nabla \rho}{\|\nabla \rho\|}
$$

and $N_{a}$ is the interpolation function associated with node $a$. It is important to note that, the local length $h$ is defined automatically taking into account the directions of high gradients and spatial discretization domain. Generally, the parameter $\beta$ is set as $\beta=1$ for smoother shocks and $\beta=2$ for sharper shocks. The compromise between the $\beta=1$ and $\beta=2$ selections as was defined in $[6]$ we consider the following average expression for $\delta_{h}^{\mathrm{NMV}}=\frac{1}{2}\left(\left.\delta_{h}^{\mathrm{NMV}}\right|_{\beta=1}+\left.\delta_{h}^{\mathrm{NMV}}\right|_{\beta=2}\right)$.

The time integration schemes considered assume that the resolved coarse scale advances in time by second order approximation and the unresolved scale can advance by first and second order approximations. In the numerical experiments we considered the following notation: NMV-FP (or DD-FP) for first order and NMV-SP (or DD-SP) second order approximations. 


\section{$3 \quad$ Numerical Experiments}

In this section we present numerical experiments considering two well known 2D benchmark problems: 'oblique shock' and 'blast wave/explosion', discretized by unstructured triangular meshes using Delaunay triangulation through the software Gmsh ${ }^{6}$. First problem used GMRES with 5 vectors to restart, tolerance equal to $10^{-5}$, the number of multicorrections fixed to 3 , the time-step size is $10^{-3}$ and the simulation is run until 3000 steps. Second problem used GMRES with 30 vectors to restart, tolerance equal to $10^{-5}$, the number of multicorrections fixed to 3 , the time-step size is $10^{-3}$ and the simulation is run until 250 steps. We compare the NMV method with the DD method. The tests were performed on a machine with an Intel Core i7-4770 3.4 GHz processor with 16GB of RAM and Ubuntu 12.04 operating system.

\subsection{D Oblique Shock Problem}

The first problem is a Mach 2 uniform flow over a wedge, at an angle of $-10^{\circ}$ with respect to a horizontal wall. The solution involves an oblique shock at an angle of $29.3^{\circ}$ emanating from the leading edge of the wedge, as shown in Fig. 2(a). The computational domain is a square with $0 \leq x \leq 1$ and $0 \leq y \leq 1$. Prescribing the following inflow data on the left and top boundaries results in a solution with the following outflow data: inflow $(\rho, u, v, p)=\left(1.0, \cos 10^{\circ},-\sin 10^{0}, 0.17857\right)$ and outflow $(\rho, u, v, p)=(1.45843,0.88731,0.0,0.30475)$. Four Dirichlet boundary conditions are imposed at the left and the top boundaries, the condition $v=0$ is set at the bottom boundary, and no boundary condition is imposed at the outflow (right) boundary.

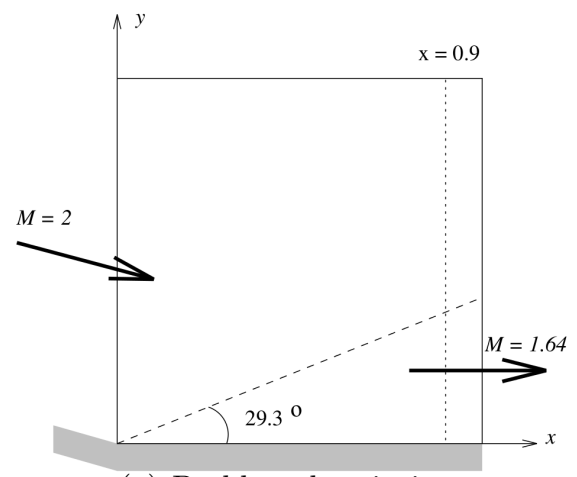

(a) Problem description.

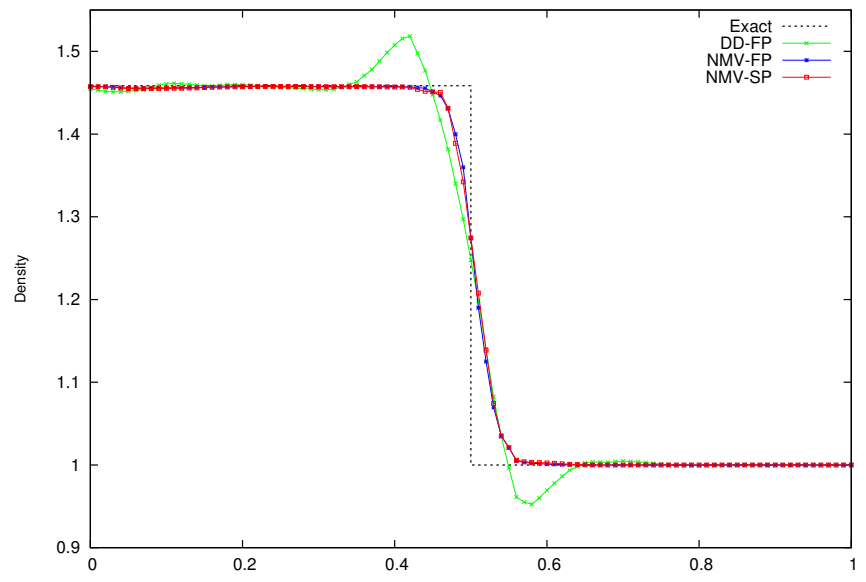

(b) Density profiles at $x=0.9$.

Figure 2: Problem description and density profile along $x=0.9$ obtained using DD-FP, DD-SP, NMV-FP and NMV-SP schemes, with the reference solution - 2D Oblique Shock Problem

\footnotetext{
${ }^{6} \mathrm{Gmsh}$ : A 3-D finite element mesh generator with built-in pre- and post-processing facilities.
} 
For all simulations we consider an unstructured mesh consisting of 1,676 nodes and 3,202 elements. Figure 2(b) shows the density profile along $x=0.9$, obtained with DDFP, NMV-FP and NMV-SP methods. The solution obtained with the DD-FP present oscillations on the left and right of the shock, whereas the solution obtained with the NMV method is more accurate and requires less gmres iterations (Table 1) than the DD method. Additionally, NMV-FP and NMV-SP are indistinguishable. It is important to note that the convergence is not achieved by the DD-SP scheme.

Table 1: Computational performance - 2D Oblique Shock Problem.

\begin{tabular}{|c|c|c|}
\hline Methods & GMRES Iterations & CPU Time (s) \\
\hline DD-FP & 129,636 & 366.116 \\
\hline DD-SP & - & - \\
\hline NMV-FP & 111,910 & 325.875 \\
\hline NMV-SP & 100,562 & 238.815 \\
\hline
\end{tabular}

\subsection{D Explosion Problem}

We consider the explosion problem for an ideal gas with $\gamma=1.4$ as described by [9]. The 2D Euler equations are solved on a $2.0 \times 2.0$ square domain in the $x y$-plane. The initial condition consists of the region inside of a circle with radius $R=0.4$ centered at $(1,1)$ and the region outside the circle, see Fig. 3(a). The flow variables are constant in each of these regions and are separated by a circular discontinuity at time $t=0$. The two constant states are chosen as inside the circle $(\rho, u, v, p)=(1.0,0.0,0.0,1.0)$ and outside the circle $(\rho, u, v, p)=(0.125,0.0,0.0,0.1)$.

For all simulations we consider an unstructured mesh consisting of 13,447 nodes and 26,492 elements. A reference solution was used considering a fine mesh with $1000 \times 1000$ computing cells by WAF method as described in [9]. Figure 3(b) compares the radial variations of the density, obtained with DD-FP, DD-SP, NMV-FP and NMV-SP schemes. In general the solution obtained by second order approximation for the unresolved scale is more accurate than the solution obtained by first order for both methods (DD and NMV). The solutions obtained with the DD-SP and NMV-SP schemes are similar, but the solution given by the NMV-SP is slightly more accurate than the solution by the DDSP. Figure 4 shows the 3D density solutions, obtained with DD-FP, DD-SP, NMV-FP and NMV-SP schemes. Although the DD method requires less GMRES iterations than the NMV method (Table 2), the NMV method provides high quality global solutions, as we can see in Fig. 4.

\section{Conclusions}

In this work we evaluate two multiscale methodologies to solve compressible flows problems: Dynamic Diffusion (DD) and Nonlinear Multiscale Viscosity (NMV) methods, 


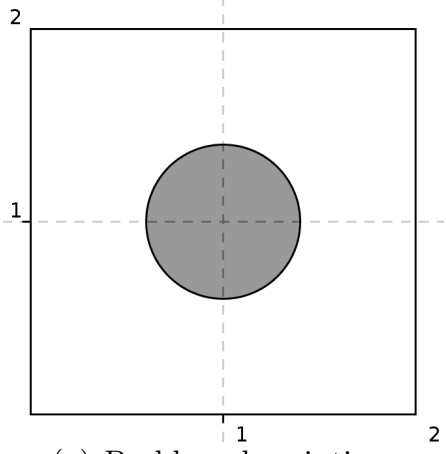

(a) Problem description.

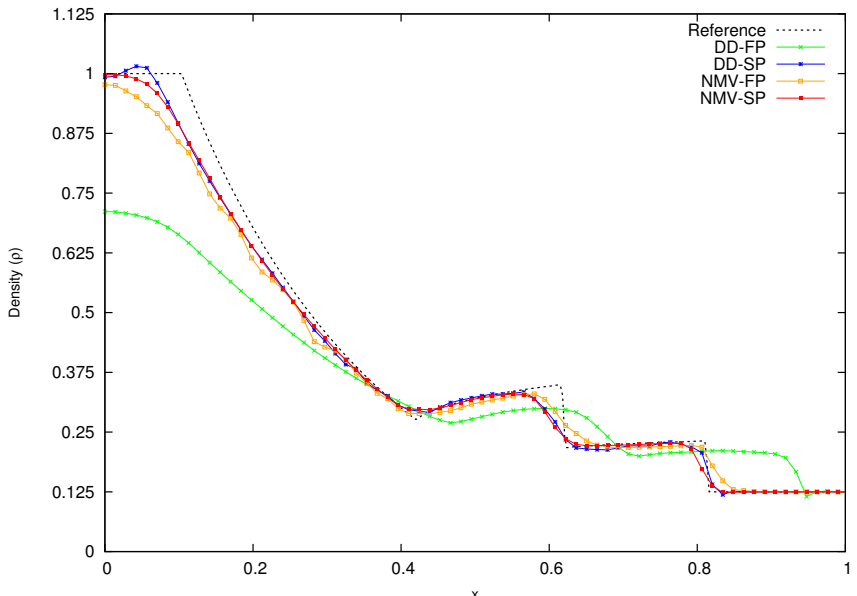

(b) Density profiles.

Figure 3: Problem description and comparisons of radial variations of density obtained using DD-FP, DD-SP, NMV-FP and NMV-SP schemes, with the reference solution - 2D Explosion Problem.

Table 2: Computational performance - 2D Explosion Problem.

\begin{tabular}{|c|c|c|}
\hline Methods & GMRES Iterations & CPU Time (s) \\
\hline DD-FP & 8,819 & 220.634 \\
\hline DD-SP & 8,446 & 176.954 \\
\hline NMV-FP & 13,087 & 298.860 \\
\hline NMV-SP & 11,669 & 220.318 \\
\hline
\end{tabular}

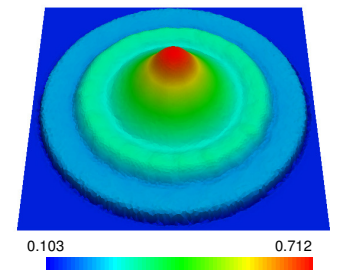

(a) DD-FP

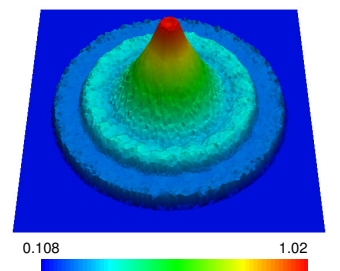

(b) DD-SP

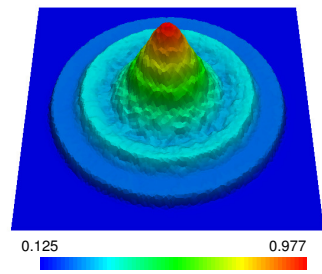

(c) NMV-FP

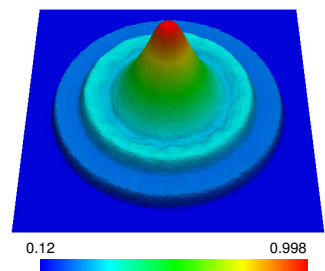

(d) NMV-SP

Figure 4: Density distribution 3D solution at time $t=0.25$. 
where the subgrid scale space is defined using bubble functions whose degrees of freedom are locally eliminated in favor of the degrees of freedom that live on the resolved scales. The performance and accuracy comparisons conducted based on the 'oblique shock' and 'blast wave/explosion' problems show us that the NMV method clearly shows more robustness than the DD method. Additionally, the NMV method provides global solutions more accurate.

\section{Acknowledgment}

This work has been supported in part by CNPq, CAPES and FAPES.

\section{References}

[1] T. J. R. Hughes. Multiscale phenomena: Green's functions, the dirichlet-to-neumann formulation, sugrid scale models, bubbles and the origin of stabilized methods. Comput. Methods Appl. Mech. Eng., 127:387-401, 1995.

[2] N. C. B. Arruda, R. C. Almeida, and E. G. D. do Carmo. Dynamic diffusion formulations for advection dominated transport problems. Mecânica Computacional, 29:2011$2025,2010$.

[3] I. P. Santos and R. C. Almeida. A nonlinear subgrid method for advection-diffusion problems. Comput. Methods Appl. Mech. Eng., 196:4771-4778, 2007.

[4] R. Z. Sedano, S. S. Bento, L. M. Lima, and L. Catabriga. Predictor-multicorrector schemes for the multiscale dynamic diffusion method to solve compressible flow problems. CILAMCE2015, November 2015.

[5] S. S. Bento, L. M. Lima, R. Z. Sedano, L. Catabriga, and I. P. Santos. A nonlinear multiscale viscosity method to solve compressible flow problems. Submitted for 16th International Conference on Computational Science and Applications, 2016.

[6] T. E. Tezduyar and M. Senga. Stabilization and shock-capturing parameters in SUPG formulation of compressible flows. Comput. Methods Appl. Mech. Eng., 195(1316):1621-1632, February 2006.

[7] A. C. Galeão and E. G. D. Carmo. A consistent approximate upwind petrov-galerkin method for convection-dominated problems. Comput. Methods Appl. Mech. Eng., 68:83-95, 1988.

[8] F. Shakib, T. J. R. Hughes, and Z. Johan. A new finite element formulation for computational fluid dynamics: X. the compressible euler and navier-stokes equations. Comput. Methods Appl. Mech. Eng., 89(1):141-219, 1991.

[9] E. F. Toro. Riemann solvers and numerical methods for fluid dynamics: a practical introduction. Springer Science \& Business Media, 2009. 